\title{
Trends and Effects of Learning through AR-Based Education in S-Korea
}

\author{
Mi-Young An, Han-Seul Kim, and Ji-Won Kang
}

\begin{abstract}
The purpose of this study is to help teachers of AR-based education by reviewing the literature on the types, learning effects and limitations of AR technology used in Korean AR-based education. Therefore, this thesis examines the literature focusing on thesis and articles of Korean thesis, and defines the definition of $A R$, technical elements, implementation method, utilization media, usage and trends, educational status, and educational effects and limitations. As a result, the classes using AR-based education could be easily immersed by learners and the presence of learning occurred. In addition, it is possible to take advantage of the AR, enabling immediate training using GPS and collaborative training using the network. AR-based education is becoming increasingly popular according to the use of digital textbooks through AR technology and AR education contents using mobile devices. However, there are limitations to AR implementation media such as qualitative differences in AR educational contents, high price, motion sickness and dizziness of HMD devices. Therefore, in order to achieve AR-based education in educational institutions and educational sites, this paper presents the development of AR implementation media suitable for the level of learners, provides flexible AR curriculum guidelines, industry-academic cooperation between companies for technology development, and inspection / censorship of educational contents. It suggests that a procedure should be provided.
\end{abstract}

Index Terms-Augmented reality (AR), AR-based education, AR-based educational contents, AR-based learning.

\section{INTRODUCTION}

With the development of advanced science and technology, it is an era of perceiving the surrounding environment and receiving knowledge information through smartphones or artificial intelligence devices. In this era, AR (Augmented Reality) has become one of the most rapidly gaining technologies due to the development of various IT technologies, the introduction of the Ubiquitous environment, and the popularization of smartphones, and has been recognized as a new technology that will lead the future IT market [1].

$\mathrm{AR}$ is a technology that allows users to interact in real time by mixing real world and virtual information [2]. A good example of this is the 'Pokemon GO' Which hit the world a few years ago. The fact that virtual characters live in the real world made the gamers interesting, and "Pokemon GO" was loved by many people. As in the above case, AR grew rapidly and various services and related markets were formed [3]. Trends in the AR market are confirmed by well-known

Manuscript received March 19, 2020; revised July 2, 2020.

Mi-Young An is with Department of Early Childhood Education at Cheju Halla University, South Korea (e-mail: myahn.ange@gmail.com).

Han-Seul Kim and Ji-Won Kang are with Cheju Halla University, Jeju, South Korea (e-mail: seul157@daum.net,ko3619@naver.com). companies in Silicon Valley. This is evidence of the fact that Apple, Facebook, Google, etc., actively discovered M\&A after discovering the possibility of AR development. In addition, it demonstrates the potential of AR's enormous potential [4]. AR is currently expanding into a variety of areas, including games, film, medical, military and distribution, as well as active research in education. According to Lee Ji-hye's (2019) study, education showed the highest rate of $13.4 \%$ in patent applications related to AR [5]. The use of AR in educational fields is increasing as AR technology is able to be useful in AR education, such as learning that is difficult for learners to experience and observe, learning details that are difficult to understand in writing, learning concepts that are not specific, and experiments that have risk factors or high costs can be applied to AR education [6]. As a result, the teaching method using AR technology is gradually increasing with the advantage of increasing the immersion of learning and direct interaction with learners.

Therefore, this paper is composed as follows to improve the quality of Korean AR-based education. First of all, the definition of $\mathrm{AR}$ and the background of appearance will be discussed, followed by the description of AR, its implementation method, and utilization media. The current research data in Korea is used to identify AR usage and education. Finally, the educational effects and limitations are stated.

\section{THEORETICAL BACKGROUND AND AR TECHNOLOGY AND TRENDS}

\section{A. AR Definition and Background}

In modern society, new technologies are being studied due to the development of IT technology and the popularization of mobile. Among them, contents that combine AR technology have been studied. For example, the Pokemon Go! is appeared in the introduction. AR stands for 'Augmented Reality' and refers to a technology that allows users to see the real environment and the virtual environment at the same time by converging the real environment in which the user lives and the virtually produced environment [7]. Commonly mentioned technologies with AR include VR and MR. First of all, VR is an abbreviation of Virtual Reality, which means to implement a situation and environment that does not actually exist [3]. MR stands for Mixed Reality, which means combining $\mathrm{AR}$ and $\mathrm{VR}$ technology to mix virtual environment with real space [8].

Azuma (1997) defined AR as a technology that provides an environment that allows users to interact with users in real time by seamlessly mixing real and virtual worlds, and 
provides users with higher realism and immersion [9]. $\mathrm{He}$ divided the characteristics of AR into three. First, AR provides a combination of virtual information reproduced by a computer in the real space. Second, the virtual information existing in the AR is presented in a coordinated manner with the real space, location and contents. Third, the information provided in the AR can interact with the user in real time [10]. In other words, AR refers to a technology capable of interacting with a user by combining virtual information in a real space in Fig. 1.

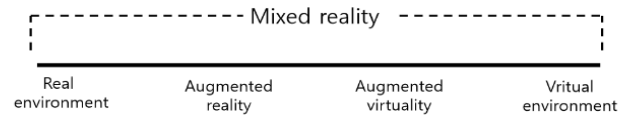

Fig. 1. Milligrams real and virtual continuity [11].

AR differs from Augmented Virtuality, which provides real-world information to the virtual world. Since the complete construction of the virtual world is difficult at present, it is AR to add virtual information based on the real situation [12].

Looking at the background of AR, the term AR originated from the novel The Master Key by L. Frank Baum as follows.

In this book, there is a pair of glasses called "Character Marker." Whenever I meet people wearing these glasses, I see a character on the forehead that tells the person's personality. Although it is a fictional story created by the artist, the concept is similar to the concept of AR, a new technology we are focusing on, in that it provides additional information of a person through glasses by combining virtual space with the real world [13]. The person who used the word AR in earnest is known as Tom Caudell. In 1990, a researcher at Boeing, he developed a wearable AR device that made it easier to maintain aircraft. The background of the word AR was used to support aviation maintenance, but $\mathrm{AR}$ is currently used in various fields as well as aviation maintenance.

\section{B. AR Technology}

Various technologies are required for $\mathrm{AR}$ contents to provide high satisfaction to users. Key technologies for implementing AR are shown in Fig. 2.

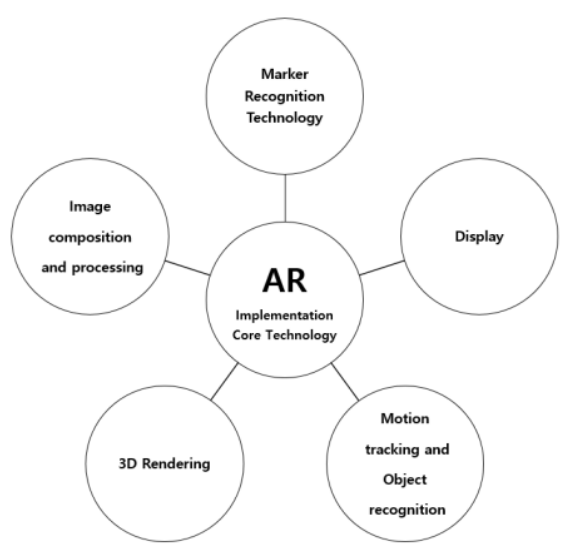

Fig. 2. AR implementation core technology.

Marker recognition technology recognizes markers in camera images after using a rectangular marker that is easy to track. After that, the camera's posture and position are calculated [2]. In order to produce a marker, the camera takes a black-and-white image and turns it into a black square. After that, the similarity is compared with the previously recorded Marker image pattern, and the vertex position of the rectangular area is used to locate the marker and obtain the coordinates [14].

Motion tracking and object recognition technologies enhance the completeness and variety of AR content by recognizing and tracking the user's actual location [15]. Lee Duk-woo [16] mentioned that it is important to grasp the location of real space, the location of real objects, and features in order for AR content to provide users with high realism and immersion. In order to achieve the localization and recognition, Tracking technology [3] and 'Feature Extraction and Recognition' technology, which tracks biometric data such as user's head, body, and eye movement in real time, are required.

Rendering refers to a technology for generating an image that can be viewed by a user through an AR browser [17]. In summary, Rendering is a technology that mixes real-world and virtual information and is important for users to use natural AR contents. Currently, the rendering technology that is being used is researching the "image-based lighting method" that estimates and renders the information from the color reflected by the object, and uses the rendering method that estimates the lighting using a 3D depth camera [15].

Image synthesis technology refers to a technology that naturally matches virtual objects in the real space [16]. Since humans are susceptible to misalignment due to inconsistency between real space and virtual objects, it is important to accurately match the three-dimensional coordinates of the real environment and virtual objects [18]. However, when connecting virtual objects to the real world, various errors such as static errors, dynamic errors, and rendering errors may occur. To resolve these problems, the visual-based technique is used, and the 3D position sensor and the camera calibration equipment are used as to reduce the error [19].

Display technology is divided into HMD (Head Mounted Device), which is worn on the head, and hand-held HMD, Projector, which is held in the hand such as non-HMD, TV, monitor, etc [20]. The related contents are shown in Table I below.

TABLE I: TYPE OF DISPLAY TECHNOLOGY

\begin{tabular}{|c|c|c|c|c|}
\hline Sortation & HMD & Projector & $\begin{array}{l}\text { normal } \\
\text { Display }\end{array}$ & Hand-held \\
\hline Panel & Ocular LCD & Projector & TV, Monitor & LCD \\
\hline $\begin{array}{l}\text { Sense of } \\
\text { immersion }\end{array}$ & High & Neutral & Low & Low \\
\hline $\begin{array}{l}\text { Wearing } \\
\text { Sensation }\end{array}$ & $\begin{array}{l}\text { Inconvenience } \\
\text { to wear for a } \\
\text { long time }\end{array}$ & $\begin{array}{l}\text { Not } \\
\text { wearing }\end{array}$ & Not wearing & Good \\
\hline Portability & $\begin{array}{l}\text { A trend that is } \\
\text { getting easier }\end{array}$ & Impossible & Discomfort & Excellent \\
\hline $\begin{array}{l}\text { Optical } \\
\text { mixing } \\
\end{array}$ & $\begin{array}{l}\text { Partial } \\
\text { possible }\end{array}$ & Possible & Impossible & Impossible \\
\hline Strengths & $\begin{array}{l}\text { A high sense } \\
\text { of immersion }\end{array}$ & $\begin{array}{l}\text { Actual } \\
\text { object } \\
\text { projection }\end{array}$ & $\begin{array}{l}\text { Be easy to } \\
\text { develop }\end{array}$ & $\begin{array}{l}\text { Be easy to } \\
\text { develop }\end{array}$ \\
\hline Problems & $\begin{array}{l}\text { May be } \\
\text { uncomfortable } \\
\text { to wear }\end{array}$ & $\begin{array}{l}\text { Shade } \\
\text { generation }\end{array}$ & $\begin{array}{l}\text { Discrepancy } \\
\text { between line } \\
\text { of sight and } \\
\text { screen }\end{array}$ & $\begin{array}{l}\text { small } \\
\text { screen }\end{array}$ \\
\hline
\end{tabular}




\section{A. AR Implementation Method and Media}

AR is a technology that combines reality and virtuality. Therefore, the natural interaction between reality and augmented objects is an important problem [21]. To resolve this problem, interaction and interface technologies are needed. This technology is meant to help perceive, and input content in VR / AR [3]. Hwang Jae-in (2018) [21] classified $\mathrm{AR}$ intraction and interface technologies into Tangible Interaction, Browsing Interface, Haptic Interaction, and Bare Hand Interaction. Tangible Interaction is an interface that manipulates digital information using physical objects. Browsing Interface is a way of matching virtual information with real images and simply looking at the data. Haptic Interaction is a way to make people feel that they are present by giving them the feeling they are actually touching when they touch a non-existent object in AR. Finally, Bare hand Interaction is the most natural and intuitive method of direct interaction with bare hands in AR environment. What the four technologies have in common is a natural connection between real and virtual objects. An example of this is the movie Iron Man 3. In the movie scene, the main character embodies AR before his parents die. In the world embodied in $\mathrm{AR}$, the protagonist shows a natural appearance without any disagreement with the actual person. Like the scenes in the movie, natural interactions in AR can further develop AR technology and will act as a non-rejection to everyone.

In order to implement $\mathrm{AR}$, a utilization medium is required. With the advancement of technology, PC and hardware have become commonplace, and AR devices have also been developed for various and simple use [22]. Types of AR devices are HMD, Projector, Non-HMD and Hand-held, as shown in the previous table.

HMD stands for "Head Mounted Device," and refers to equipment used by mounting on the head. HMD can be divided into two technical aspects. Optical see-through for viewing the real environment and virtual scene at the same time, and Video see through for showing the result at the same time by combining the video taken by the camera with the virtual world at the same time [20].

Projector can select and touch the augmented contents by using various objects, spaces, human hands, tools, etc. instead of the mouse, and can operate as naturally and easily as the real environment. This has the advantage that a user can use a program utilizing AR even without using a dedicated device. However, there is a disadvantage that the use is restricted due to the fixation of the different position depending on the brightness of the surrounding environment and the size of the projector [23].

Non-HMD stands for 'Non-Head Mounted Device' and means equipment that is not worn on the head. Non-HMD is divided into small and large display equipment. Small display devices are often used in environments such as medical applications where it is difficult to wear heavy HMDs and require high resolution images. Large display equipment is used in offices and flight simulators with a large number of participants [24]. Hand-held HMD means to be used in the form of a portable device, and may be an example of a device such as a smartphone, PDA, etc. [2].

\section{B. AR Status and Trend}

Nowadays, in broadcasting and various media, the term 'one-person household age' is used to describe the increase of one-person households. People use smart devices and mobile devices more than traditional media devices. In addition, the demand for VR and AR technology services is increasing due to the increasing number of people seeking convenience and enjoyment in everyday life [16].

VR and AR technologies are increasingly being applied. First, as the technology of VR and AR is expanded in the field of portable devices including smart devices and mobile devices, display development using spatial augmented reality technology including AR together with VR display technology is being made. These advances have made it possible to instantly view or easily obtain information, which can be applied to various fields such as games, healthcare, education, broadcasting, advertising, and manufacturing. This makes it easier for users to access AR. In addition, social network, military, design, and cultural arts are also using VR and AR technology to expand the scope of application [25]. An example related to this is as follows.

First of all, Hyundai Motor Group of Korea is planning to apply a new infotainment system to Genesis models. AR navigation is the biggest change in this system. AR navigation displays images taken by cameras attached to a vehicle on a monitor in real time to graphically display driving routes based on map data and vehicle motion sensors. It is expected to deliver driving information more intuitively than the existing navigation system and to reduce the mistake of the driver entering the road [26].

Bibimbble, a mixed-reality company, conducted a hologram talk concert with Baek Beom Kim-gu. A virtual reality game called "Hidden Story", which restores the stories of historical figures, and Baek Beom Kim-gu, have a photo studio using AR to take pictures. It is believed that this is embodied as a historical theme park where visitors can experience and sympathize without simply touching education [27].

AR technology has also begun to be introduced in the entertainment sector. For example, Korean company SM and overseas company Intel will show 'immersive' content by combining Volumetric Capture technology, interactive content, and AR technology with K-pop [28].

Home shopping, which can be used in everyday life, is introducing services using AR technology. Lotte Home Shopping has introduced a 'moving AR' service that allows users to experience or arrange functions of furniture or appliances virtually using AR technology. 'Moving AR service' can control the virtually produced sofa back, open the inside of the refrigerator, and play the video on TV [29].

As the application fields of AR technology are diversified, the market is being re-adjusted because technology development is lower than people's expectation due to 'convenience of use environment, expensive VR / AR media device, lack of killer contents', etc. [30]. In terms of market involvement, most software companies are the most involved, and the gaming sector is traditionally dominated by Japanese companies. However, in high-potential areas such as defense and healthcare, new software companies are trying to take the lead in the market [16].

In the AR and VR sector, according to clinical researcher Seo Kyung-won [31], the global AR / VR market is expected to reach about $\$ 115$ billion in 2022. In particular, $A R$ is 
expected to grow more than six times that of VR. This can be seen by the movement of large overseas companies. Overseas, Microsoft, Google, HTC, Sony and Facebook, which have always been the leaders in software and video technology, have discovered the potential for AR/VR and acquired and merged with companies with various content and technologies to increase the size of the market [16]. At present, however, Korea is actively developing dedicated devices and hardware mainly for large companies to provide AR and VR services, but has weak competitiveness in the development of software and content technology [16]. Although KT and SK, such as mobile carriers and developers, are promoting and investing in the business, global competition is not enough [30].

The reason why Korea's AR technology will lack global competitiveness in the future is the Korean patent application problem. Currently, the proportion of patent applications in Korea is $85.4 \%$, which is higher than in other countries. However, the share of foreign patent applications is the lowest in Korea compared to the US, China, Japan, and Europe. In order to enhance the global competitiveness of the Korean AR market, companies need to focus on overseas applications [32].

TI, Samsung, and SK Hynix, the leaders in traditional hardware, are striving to preoccupy the core components of $\mathrm{AR}$ and VR [16]. It also invests a lot in related AR / VR fields in various fields, and has given rise to an active technological competition for startups [31]. In 2019, the Korea Institute of Industrial Technology (Saengwon Institute of Science and Technology) developed a method to solve the long-term immersion difficulties and dizziness, which were obstacles to the popularization of AR and VR. It is difficult for users to immerse for a long time because the display of AR / VR devices is darker and lower in clarity than smartphones and TVs. In order to compensate for this, Dr. Kwan-Hyun Cho, a member of the micro-nano process group, has developed a high-resolution OLED display manufacturing process suitable for VR / AR. This technology enables the mass production of high-resolution AR / VR displays at low cost, and can also widen the viewing angle of the screen. AR / VR display using OLED technology can increase user's immersion and reduce dizziness [33].

As such, AR / VR is expected technology and will affect active industry. On the foreign side, there is Apple. Apple is launching smart glasses with AR capabilities in 2023. Unlike current devices, it is a device that can be used lightly like glasses. As AR functions are added, various digital information and contents projected on the surrounding environment will be seen [34]. Soon the VR / AR seen in the movie will become reality.

\section{EDUCATIONAL EFFECT OF AR}

\section{A. Status of Educational Use of $A R$}

AR technology is a situation where various world-class companies and research teams are making various investments with interest in implementing AR technology and services [16]. In particular, educators in Korea and abroad are interested in the educational effects of AR, and research, produce, and utilize various educational contents using AR. In this study, the educational use status of AR was divided into $\mathrm{AR}$ education contents using digital textbooks and mobile devices in Table II.

First, the use of digital textbooks, a team of professors Kim Jung-hyun of the Department of Computer Science and Engineering, Pohang University in Korea received the grand prize at the 2004 Computer Graphics International Conference in the United States. The AR works developed by Professor Kim's team showed that they made a race of rabbits and turtles in Aesop's fables by hand-operating system [35]. This shows the beginning of AR technology using books in Korea.

In 2007-2009, digital textbook commercialization began and the Digilog Book appeared. The Ministry of Education and Human Resources Development announced that in 2007, digital textbooks will be developed to commercialize digital textbooks [36]. The pilot school was expanded to 100 elementary, middle, and high schools by 2012, and the plan for full expansion was examined from 2013. The Gwangju Institute of Culture and Technology presented the Digilog Book, which combines paper and e-books. Digilogbook, the first of its kind in Korea, is an "realistic interactive ubiquitous book" designed based on AR. This shows the applicability of edutainment in providing education and entertainment simultaneously [37].

In 2008, KERIS and Daejeon Metropolitan Office of Education established a virtual learning environment for elementary and secondary schools, discovered and applied operational models, and developed VR / AR-based experience-based English learning contents [38]. Hanul Neotek has developed an AR application business model. It shows the growth of educational contents and AR market, and the emergence of AR-based fairy tales [39].

Daejeon Office of Education developed augmented reality education contents and systems such as Korea Electronics and Telecommunications Research Institute (ETRI), Korea Education and Research Information Service (KERIS), and applied them to English, mathematics, social and science classes at Crystal Elementary School since 2009. At this time, we used learning materials using markers [40].

Since the 2010s, AR technology has been used in collections and learning books, and digital textbooks have been promoted in earnest. In 2011, the Samsung Party developed an AR book that can be used as a smart device in cooperation with Samsung Electronics. It is possible to watch and enjoy lively digital animation with smart devices along with a booklet [41]. In 2012, Woongjin Thinkbig released its first collection of AR sciences [42]. The adoption of AR provided three-dimensional scientific information that could not be felt in existing books. In 2013, in the future, publishers were able to use AR services in their preparations for middle and high school students [43]. Since then, AR service has been implemented in elementary school reference books [44].

In 2015, DIPSEE launched Animal Theme Photo Book, Play with Animals, for children. The AR application "Lime AR" will scan the mark inside the photobook to reproduce actual images, sounds, and introductory voices of professional voice actors. Through this, it is characterized by enhancing the learning effect by inducing the child to feel the animal intimately [45]. We can see the possibility of applying 
AR technology education from middle school students to young children.

TABLE II: AR UTILIZATION EDUCATION STATUS BY YEAR

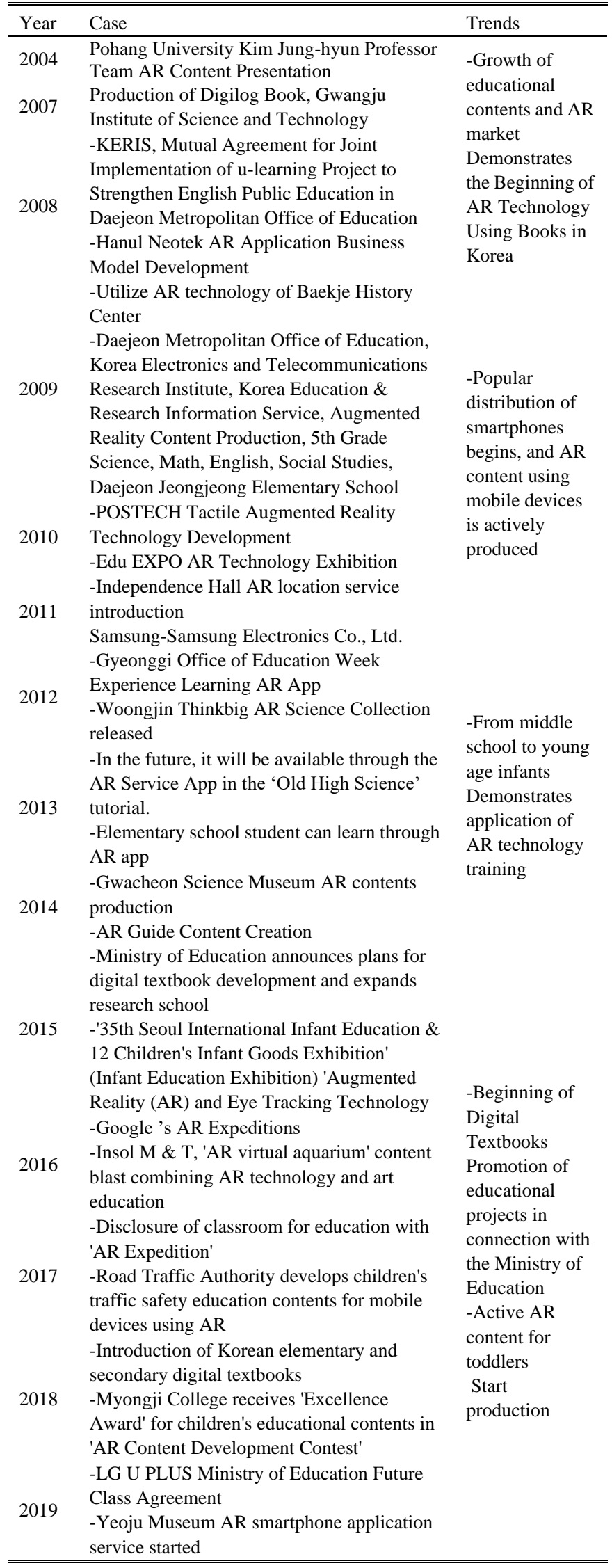

From 2016 to 2019, digital textbooks were introduced, and the future education class was signed with the IT companies and the Ministry of Education. Since 2018, digital textbooks using VR and AR have been introduced in social, science and English textbooks for elementary school students in grades 3-4 and secondary school [46]. In order to provide high quality instruction using digital textbooks, local school districts are conducting relevant training for teachers [47]. In fact, in Anyang Heesung Elementary School, there were cases where parents open classes and training sessions were conducted using AR digital textbooks [48].

In addition, in 2019, the Ministry of Education, Microsoft Korea, and LG Uplus signed a business agreement to build a "future classroom using AR / VR." The term 'future classroom' refers to a realistic educational model that uses $\mathrm{AR}$ and VR to learn field trips and hands-on learning. In 'Future Classrooms', AR/VR content 'Talk Talk Experience Class' and Google's AR/VR service 'Expeditions' can be used to observe cultural assets, plants and local attractions that were not seen as a matter of time and space [49].

AR content using mobile devices is being actively produced around 2010, when smartphones began to be popularized. The 13th " Edu Expo " in 2010 is content that uses AR to meet the changing e-learning environment [50]. This is an example of the possibility that AR content can be applied in a classroom environment. An example of using a smartphone is the Independence Hall application. The Independence Hall has launched the "Independence Hall Smartphone Application" service since 2011. The application is provided with a system that uses GPS to guide the location of key amenities [51]. Learners can enjoy the Independence Hall as a guide through AR location-based services.

In 2012, the Gyeonggi-do Office of Education developed the application of 'Creative Experience Learning'. 'Creative experiential learning' uses the GPS to guide the learner's desired course to the AR screen. Since the first visited place can be easily moved, the creative experience activity can be enhanced [52]. In 2014, the Gwacheon Science Museum established an exhibition hall where the AR system, "Does it happen every day," was observed [53]. The Ministry of the Future and the Cultural Heritage Administration developed an AR application that introduces Korea's old palace [54]. In early 2015, various companies presented educational contents using AR, demonstrating the feasibility of AR in early childhood education [55]. Insol M\&T has produced AR virtual aquarium solution for toddlers to create contents for toddlers to enjoy coloring through marker technology [56]. AR contents for children including AR safety education contents produced by transportation authorities have been delivered additionally on the solution [57]. The R-eve team of Myeongji University can expect to develop AR education contents of various ages in the future, such as receiving contents for children's educational contents at 'VR EXPO 2018' [58]. Overseas companies are also producing various contents to apply AR to education. An example is Google's "Expeditions", which was released in 2016. The learner who uses expedition learns by watching the smartphone in the classroom and experiences unobservable contents such as hurricane blowing and volcanic eruption [59].

An example of AR use in the United States is Magic Leap's AR project. Students will experience life through this project, such as observing whales jumping out of the basketball court. The Wiscon College of Medicine, Madison, USA produced the documentary "Dow Day" using AR. "Dow Day" allows 
students to see what happened at their location [60].

The development of digital textbooks and the development of AR educational contents using mobile devices were surveyed in this section. AR-based education will be developed in the future by adding high technology and universality.

\section{B. AR Education Effects and Limitations}

Today, teachers are studying teaching methods that use a variety of materials and media to provide education that will inspire students and enhance the effectiveness of learning. In that sense, the emergence of AR has led to the direction of new education for educators studying the education media.

In this study, the educational effect of AR was analyzed based on observation learning type, experimental activity type, mobility-based type, and cooperative learning type.

The effect of AR-based learning is, firstly, the presence of learning by context awareness [61]. Related examples include classes that explain the structure, function, and use of fire extinguishers. AR provides learners with a virtual fire environment and a virtual fire extinguisher. This makes it easy for learners to understand why a fire extinguisher is used and how to use it in the context of extinguishing a fire using a fire extinguisher. This class belongs to the observation type learning type, which is a learning type that presents a specific object to the screen using the AR technique and then suggests the name, function, and operation of each part of the object [22]. In other words, the use of AR content goes beyond mere conception to achieve a meaningful application effect in the actual context [62], creating the context of learning and raising the learner's presence.

Second, in the process of the learner leading the operational activities through the AR, the ability to easily understand the contents of learning and to reconstruct the knowledge voluntarily is enhanced [61]. In addition, AR technology that seamlessly mixes real and virtual worlds provides users with enhanced realism and immersion [63]. In summary, AR has no space constraints and allows users to voluntarily reconstruct knowledge. The home of the class in this regard is the activity of making your own city. Through $\mathrm{AR}$, we create a virtual city, learners build autonomously, and perform various and complex manipulation activities. This is similar to the experimental activity type, which is an implementation method in which the learner can operate the operation through AR after creating a virtual space using AR technology [22].

Third, in the case of educational content using a mobile base among AR technologies, immediate lessons are possible depending on the location of the learner. An example of a mobility-based type is Daewoo E\&C's Prugio Complex AR experience service. Through the GPS reception of the learner, it provides the service to identify the location and find the description in the complex and listen to the explanation [64]. In other words, when the learner carries and moves the AR implementation medium, the AR education content provides the learner with appropriate learning contents according to the location [21].

Fourth, social cooperativeness is obtained through cooperative learning of AR-based learning [61]. AR-based learning communicates with a network. Solving learning tasks together in a network together, learners can achieve social cooperation through division of labor and cooperation that share roles. Mark Billinghurst said that the advantage of AR education contents is that it uses smartphones, tablet PCs, etc., which can be easily used in the daily life of users, and gives a new metaphor of Tangible interface [22]. It seems to be related to collaborative learning activities, which are a type in which AR is used to solve a learning task in the process of linking and communicating with a network of learners.

While AR-based education has an educational effect, it also reveals educational limitations.

The educational limitations are as follows: First, AR education contents in Korea have a wide quality gap between contents [65]. Currently, there are data on dinosaurs in the digital textbooks provided to elementary schools. Dinosaurs' behavior in digital textbooks is dynamic, but there is no sound, making them less immersive. The reason is that there is no scientific basis to prove how non-existent dinosaurs make sounds, so they can't put sounds. Unlike educational contents and cartoons, which are made in the private sector, digital textbooks cannot contain unfounded materials because of textbooks [66]. There is a concern that learners may be confused due to the severe gap between AR educational contents.

Second, it is difficult to build an AR education platform and it is difficult to develop AR contents due to high development cost [67]. Due to cost concerns, educational institutions are not introducing new technologies. Most educational institutions lack budget and do not make new investments [65]. Currently, Korea's 4th Industrial Revolution policy support is the lowest compared to the US, China, Germany, and Japan. However, the regulation of the 4th Industrial Revolution is the highest and there is a lack of support for AR education [68].

Third, problems with the user's health occur when wearing the HMD device [69]. When wearing an HMD device, there is a delay between the speed at which the eyes are turned and the image seen on the screen. This delay can lead to a user's cyber sickness and, if sustained, can lead to panic, epilepsy and photosensitivity attacks [70]. In response to this concern, the DOE wrote to elementary schools nationwide to refrain from using HMD devices [71]. The development of technology to solve the health problems of HMD devices is needed.

\section{CONCLUSION AND SUGGESTIONS}

This study analyzed papers related to AR technology and trends in Korean AR-based education to investigate the effects and trends of learning through AR-based education in Korea.

AR technology is a technology that naturally connects the real world with virtual information. The technologies for naturally implementing AR are classified into five categories: marker recognition technology, 3D rendering technology, motion tracking and object recognition technology, image synthesis and processing technology, and display technology. There are various interaction and interface technologies for implementing AR technology, and the implementation medium is HMD, Projector, Non-HMD, Hand-held.

AR technology is being expanded in various fields due to 
the popularity of mobile handheld devices such as smartphones and the increasing number of people seeking convenience in daily life. Representatives include games, healthcare, education, broadcasting, advertising, manufacturing, social networks, military, design, and cultural arts. This can be seen through the case of various companies in Korea and abroad. In Korea, development of dedicated devices and hardware has been actively conducted by large corporations to provide AR / VR services. However, Korean AR technology is weakly competitive in the development of software and content technologies [16]. It also shows a lack of AR patent applications. To overcome the limitations of AR technology, much research and development is required.

Looking at AR-based education in terms of digital textbooks, we can see that AR utilization education began in 2004. In 2007, the company established a policy to commercialize digital textbooks. In 2015-2016, the Ministry of Education expanded the planning and operation schools for digital textbooks. In 2018, Korea introduced digital textbooks to elementary and secondary schools. For example, in 2019, the Ministry of Education, Microsoft Korea, and LG Uplus signed a business agreement to build a " future classroom using AR / VR. "

As smartphones have evolved, the utilization of AR education contents using mobile devices has increased. In 2011-2012, GPS-based smartphone applications were used, such as 'Independent Museum Smartphone Application' and 'Creative Experience Learning'. Since 2014, as in the case of Gwacheon Science Museum, applications that allow users to observe things through AR are frequently used. Based on this, we can predict the development of education market using Korean AR contents. As in Korea, the use of AR is gradually increasing in overseas educational environments. Google's expeditions, "Magic Leap," and "Dow Day" were examples. Actively researching and utilizing AR educational contents in Korea and abroad will provide students with high quality AR educational contents.

The learning effects of learners who are provided with AR content education can be summarized as follows. First, learners understand the context of learning and gain presence through AR educational content. Second, learners gain realism and immersion through the natural connection between real and virtual worlds. Third, when AR content provides location-based services, learners can immediately educate them based on their current location. Finally, social cooperative ability can be developed through collaborative education of learners through network connection.

Using AR content in education is not only good. It should be recognized that there are limitations to using AR. Currently, AR educational contents are immature to distribute to learners. In addition, in the case of content wearing HMD devices, there are many products that are difficult on the body when used for a long time such as motion sickness and neck disk, and the financial burden is generated at high cost. In order for AR-based educational contents to be actively used in Korea, the research of technicians who want to develop more immersive contents is required, and AR-based educational contents should be developed and universalized.

AR-based education provides learners with educational effects such as immersion, presence, immediate education and cooperative education, but there are disadvantages such as performance and price of AR implementation media. This paper suggests the following to supplement this problem.

First, most AR-based educational contents currently used use mobile devices such as smartphones and tablet PCs. However, due to the occurrence of side effects, such as early childhood smartphone addiction is forming a public opinion and social atmosphere that the use of infants and toddlers smartphone is not right. It is necessary to develop alternative AR implementation media to reduce the occurrence of side effects or to develop educational contents suitable for the level of infant development.

Second, AR-based learning is used in Korean educational institutions in line with existing curriculum guidelines. In the case of developing AR-based learning according to the existing curriculum, there is a pity that AR technology cannot be utilized because only content based on facts should be entered. Thus, the effects of AR-based learning, such as immersion and presence are not achieved. In the future, AR-based learning needs to be flexibly changed in the direction that the existing curriculum guideline will have the effect of AR technology.

Third, AR is a rising technology in Korea and overseas conglomerates. This can be seen by examining the cases of mergers and acquisitions of overseas dinosaur companies and the trend of AR / VR development by large Korean companies. When developing AR-based education in the future, it is necessary to develop industry-academic cooperation with large IT companies and SMEs.

Fourth, educational AR content produced by a general company may provide information that is not accurate or educationally appropriate in order to induce learner's interest. In order to provide learners with accurate information, procedures for inspecting / censoring educational AR content should be provided.

\section{CONFLICT OF INTEREST}

The authors declare no conflict of interest.

\section{AUTHOR CONTRIBUTIONS}

An conducted the research; Kim and Kang analyzed the data; Kim and An wrote the paper, all authors had approved the final version.

\section{REFERENCES}

[1] W. H. Lee, "Classification and practical use of educational augmented reality application for mobile media," Journal Korea Society of Visual Design Forum, vol. 47, pp. 105-114, 2015.

[2] H. J. Park, T. H. Han, J. C. Jeon, and G. H. Kim, "E-learning technology trends based on augmented reality," Review of Korean Society for Internet Information, vol. 10, no. 2, pp. 12-22, 2009.

[3] J. S. Lee and J. Y. Kim, "A study on spatial characteristics of immersion and reality in cases of VR and AR technology and contents," Journal of the Korean Institute of Interior Design, vol. 28 , no. 3, pp. 13-24, 2019.

[4] I. H. Cho, "AR/VR present and future and SKT's R\&D strategy," Korea Multimedia Society, vol. 21, no. 1, pp. 23-31, 2017

[5] J. H. Lee, "A study on the revitalization of virtual reality-based education," Journal of the Korean Society Design Culture, vol. 25, no. 1, pp. 357-366, 2019

[6] H. J. Lee, S. A. Cha, and H. N. Kwon, "Study on the effect of augmented reality contents-based instruction for adult learners on academic achievement, interest and flow," Journal of the Korea Contents Association, vol. 16, no. 1, pp. 424-437, 2016. 
[7] K. H. Noh, H. K. Jee, and S. H. Lim, "Effect of augmented reality contents based instruction on academic achievement, interest and flow of learning," Journal of the Korea Contents Association, vol. 10, no. 2, pp. $1-13,2010$

[8] J. H. Song, J. H. Kim, M. H. Yoo, and Y. H. Koo, "A meta-analysis on effects of VR, AR, MR-based learning in Korea," Journal of Korean Association for Educational Information and Media, vol. 24, no. 3, pp. 459-488, 2018.

[9] J. J. Oh, "Exploring the possibility of utilization on augmented reality technology in geography learning," Journal of Geographic and Environmental Education, vol. 20, no. 1, pp. 79-94, 2012.

[10] G. Lee, "The present and future of augmented reality technology," Telecommunications Technology Association, vol. 133, pp. 88-93, 2011.

[11] P. Milgram and F. Kishino, "A taxonomy of mixed reality visual displays," IEICE Trans, Information Systems, vol. 77, no. 12, pp. 1321-1329, 1994.

[12] B. K. Kye, J. H. Kim, and J. H. Rye. (2007). Educational understanding of augmented reality. Korea Education \& Research Information Service. [Online]. Available: https://www.keris.or.kr/main/ad/pblcte/selectPblcteRMInfo.do?mi=11 39\&pblcteSeq $=11026$

[13] H. K. Cho and S. H. Kim, "A study on the edutainment contents elements in augmented reality (AR) educational contents design," Journal of the Korean Society Design Culture, vol. 25, no. 1, pp. 441-452, 2019.

[14] B. J. Sung and E. J. Choi, "Augmented reality domestic and foreign technology trend and development outlook," Korea Institute of Science and Technology Information Emerging Issue Report, vol. 16, pp. 5-41, 2007.

[15] H. J. Lee et al., "Republic of Korea expands to content!" Korea Culture Technology Magazine, vol. 48, pp. 16-18, 2016.

[16] D. W. Lee. (14 November 2018). Virtual and augmented reality applications technology trends. Institute for Information \& communication Technology Planning \& Evaluation. [Online]. 1872. pp. 2-15. Available: https://www.iitp.kr/kr/1/knowledge/periodicalViewA.it

[17] B. H. Kim, "A context-aware mobile augmented reality platform," Journal of the Korea Institute of Information and Communication Engineering, vol. 16. no. 1, pp. 205-211, 2012.

[18] J. H. Lee and H. W. Cho, "Implementation of infant learning content using augmented reality," Journal of the Korea Contents Association, vol. 15 , no. 1 , pp. $257-263,2010$

[19] Korea Copyright Commission. (29 July 2010). The present status of augmented reality technology - Focused on domestic and foreign trends. Korea Copyright Commission. [Online]. Available: http://www.copyright.or.kr/information-materials/trend/the-copyright/ view. do?brdctsno $=8610 \&$ pageIndex $=1 \&$ portalcode $04=\&$ notice $Y n=\&$ nationcode $=\& b r d n o=35 \&$ etc $1=\&$ searchkeyword $=\&$ portalcode $=04 \& b$ dclasscode $=\&$ searchText $=\% \mathrm{EC} \% \mathrm{~A} 6 \% 9 \mathrm{D} \% \mathrm{EA} \% \mathrm{~B} 0 \% 95 \% 20 \% \mathrm{ED} \% 9$ $8 \% 84 \% \mathrm{EC} \% 8 \mathrm{~B} \% \mathrm{~A} 4 \% 20 \% \mathrm{EA} \% \mathrm{~B} 5 \% \mathrm{AC} \% \mathrm{ED} \% 98 \% 84 \% 20 \% \mathrm{EA} \% \mathrm{~B}$ 8\%B0\%EC\%88\%A0\%20\%ED\%98\%84\%ED\%99\%A9\&searchTarget $=$ SUBJECT\&servicecode $=06 \&$ brdctsstatecode $=$

[20] S. Y. Kim and J. W. Lee, "A study on the experience of the exhibition design of augmented reality-based - Focusing on," Experience Module Strategic (SEMs), vol. 30, pp. 233-242, 2014.

[21] J. I. Hwang, "Types and analysis of interaction methods used in augmented reality," Korea Information Processing Society Review, vol. 25 , no. 2 , pp. 39-47, 2018.

[22] J. H. Lee, "A study on the educational use of augmented reality based mobile education content - Case analysis of mobile augmented reality application for education," Journal of the Korean Society Design Culture, vol. 24, no. 1, pp. 569-585, 2018.

[23] G. S. Heo, D. W. Lee, H. T. Jeong, and J. S. Park, "Technical trends of projection-based augmented reality," Electronics and Telecommunications Trends, vol. 26, no. 5, pp. 92-101, 2011.

[24] 4IR. (22 February 2018). Element technology related to AR (Augmented Reality) technology. 4IR. [Online]. Available: https://4ir.kisti.re.kr/ick/cmmn/viewPost/20180222000025

[25] H. Y. Kim and B. K Choi, "Study on the technical trend of augmented reality and application method of ceramic products," Korea Science \& Art Forum, vol. 19, pp. 269-280, 2015.

[26] G. H. Kim. (19 November 2019). 'The car takes care of the noise of the road, changes lanes.' Hyundai Motor Company is focusing on cutting-edge technologies in Genesis. Consumernews. [Online]. Available: $\quad \mathrm{http}: / / \mathrm{www}$. consumernews.co.kr/?mod=news\&act= articleView\&idxno $=535083$

[27] S. J. Kim. (19 November 2019). 'Torque Concert with Baekbeom Kim Koo' be realized with the technology of bibimble. Sportsseoul. [Online]. Available: http://www.sportsseoul.com/news/read/850966
[28] H. J. Kim. (14 November 2019). Views 360 degrees performance of 'NCT127'. SM-Intel, K-pop and advanced technology included. Yonhap News Agency. [Online]. Available: https://www.yna.co.kr/view/AKR20191114053600005?input=1195m

[29] J. M. Oh. (17 September 2019). Lotte home shopping, moving AR Experience and purchase in augmented reality. Hankyung. [Online]. Available: https://www.hankyung.com/economy/article/201909175554g

[30] Y. C. Jang, J. S. Im, and J. H. Kim, "Virtual and augmented reality (VR/AR) industry trends," The Journal of The Korean Institute of Communication Sciences, vol. 36, no. 1, pp. 35-41, 2018

[31] S. W. Im and G. W. Seo. (10 July 2018). KISTEP technology trend brief (AR/VR technology). Kistep. [Online]. Available: https://www.kistep.re.kr/c3/sub8.jsp?

[32] J. G. Lee. (13 November 2019). Rapidly growing 'VR, AR' foreign patents are low. Digitaltimes. [Online]. Available: http://www.dt.co.kr/contents.html?article_no=2019111402101531731 $001 \&$ ref $=$ naver

[33] J. Y. Ryu. (18 November 2019). Make 'large OLED for VR and AR' cheaper. Increase the sense of immersion and resolve dizziness. Moneytoday. [Online]. Available: https://news.mt.co.kr/mtview.php? no $=2019111810124028062 \&$ outlink=1\&ref=http $\% 3 \mathrm{~A} \% 2 \mathrm{~F} \% 2 \mathrm{Fsearch}$ .daum.net

[34] Y. S. Baek. (13 November 2019). Apple, AR smart glasses launched in 2023. AR headset for 2022. Digital Today. [Online]. Available: http://www.digitaltoday.co.kr/news/articleView.html?idxno=217401

[35] Y. J. Lee. (17 August 2004). Won the grand prize of Professor Kim Jung-hyun team of Pohang University. Yonhap News Agency. [Online] Available: https://news.naver.com/main/read.nhn?mode=LSD\&mid $=$ sec $\&$ sid $1=102 \&$ oid $=001 \&$ aid $=0000733981$

[36] Y. Y. Lee. (17 July 2016). Augmented Reality in the Classroom Introduced digital textbooks in mid-2018. Yonhap News Agency. [Online]. https://www.yna.co.kr/view/AKR20160715151600004

[37] H. Y. So. (20 November 2007). Touch, smell, and listen to books Etnews. [Online]. Available: http://www.etnews.com/200711190133

[38] S. H. Lee. (28 August 2008). KERIS, together with the Daejeon Metropolitan Office of Education, promotes English U-learning. Etnews. [Online]. Available: http://www.etnews.com/200808270237

[39] C. S. Lee. (25 November 2008). When AR meets a fairy tale book, the main characters live. Daily Newspaper. [Online]. Available: http://news.imaeil.com/NewestAll/2008112506411072378

[40] Y. K. Kim. (06 May 2010). The augmented reality has completely changed the classroom landscape. Etnews. [Online]. Available: http://www.etnews.com/201005050051

[41] S. C. Ahn. (27 December 2011). Samseongdang-Samsung electronics co., develops 3D video by smartphone for AR book. Etoday. [Online] Available: http://www.etoday.co.kr/news/view/523935

[42] M. S. Jo. (03 December 2012). First release of augmented reality science collection. Woongjin ThinkBig 'Science Picture Book Banana Rocket'. Heraldcorp. [Online]. Available: http://news.heraldcorp.com/view.php?ud=20121203000865\&md=201 21206004529_BL

[43] J. U. Kim. (28 November. 2013). <New product> Mirae-n, schoo grades study material 'Allead Higher science'. Yonhap News Agency. [Online]. Available: https://news.naver.com $/ \mathrm{main} / \mathrm{read} . n h n$ ?mode= LSD\&mid $=$ sec \& $\operatorname{sid} 1=101 \&$ oid $=001 \&$ aid $=0006620614$

[44] J. U. Lee. (19 December 2013). Popular references Mirae Co., Ltd., Augmented Reality Service. Financial News. [Online]. Available: http://www.fnnews.com/news/201312190847319434?t=y

[45] I. G. Kang. (12 January 2015). DIPSEE, animal-themed photo books for children's 'play with animals' Launch. MoneyS. [Online]. Available: http://moneys.mt.co.kr/news/mwView.php?type=1\&no $=2015011208158047334$ \&outlink $=1$

[46] J. W. Seo. (14 April 2017). Introducing digital textbooks in 2018 "Smart learning is on the rise". Edu Donga. [Online]. Available: http://edu.donga.com/?p=article\&ps=view\&at_no=201704141048343 44702

[47] I. H. Jo. (22 November 2019). Gyeongbuk office of education, digital textbook application and smart education training. News Services Provider. [Online]. Available: http://www.nspna.com/news/?mode=view\&newsid=395377

[48] J. S Park. (07 October 2019). Anyang heesung elementary school, parents' digital textbook open classroom. Kyeong Gi Ilbo. [Online]. Available: https://www.kyeonggi.com/news/articleView.html?idxno=2174789

[49] G. T. Lee. (23 October 2019). ICT / Media LG Uplus builds future classes using AR and VR. Chosun Biz. [Online]. Available: https://biz.chosun.com/site/data/html_dir/2019/10/23/2019102300644 .html 
[50] T. H. Hwang. (31 March 2010). 'Edu Expo' has just risen. Etnews. [Online]. Available: http://www.etnews.com/201003300280

[51] B. H. Kim. (11 October. 2011). Release of the independence hall mobile app. Ccdailynews. [Online]. Available: http://www.ccdailynews.com/news/articleView.html?idxno=237280

[52] J. Y. Yun. (04 April 2012). Gyeonggi office of education launches weekend experience learning mobile app. Sedaily. [Online]. Available: https://news.naver.com/main/read.nhn?mode=LSD\&mid $=$ sec \&sid $1=102 \&$ oid $=011 \&$ aid $=0002229790$

[53] J. O. Jeong. (21 April 2014). How is my blood. Gwacheon Science Museum 'Blood Journey'. The Asia Business Daily. [Online]. Available: http://view.asiae.co.kr/news/view.htm?idxno=2014042109461828730

[54] H. W. Kim. (26 January 2014). Old palace on smartphone 'SSOG' YTN. [Online]. https://www.ytn.co.kr/_ln/0102_201401260003534400

[55] J. H. Choi. (22 July 2015). August 13 early childhood education opening. Introducing the latest education technology. Korea Business News Co., Ltd. [Online]. Available: https://news.naver.com/main/read.nhn?oid=215\&aid=0000337828

[56] J. H. Jeong. (13 January 2016). Insol M \& T, 'AR virtual aquarium' content blast combining AR technology and art education. Etnews. [Online]. Available: http://www.etnews.com/20160113000296

[57] J. Y. Choi. (28 December 2017). Development of child safety education contents using AR technology. Munhwa.com. [Online]. Available: 2017122801071103025001

[58] J. Y. Lim. (28 December 2018). Myongji college Wins 'AR content development contest'. UNN. [Online]. Available: http://news.unn.net/news/articleView.html?idxno=205233

[59] Expeditions service center. What is Expeditions? Expeditions. [Online].

Available: https://support.google.com/edu/expeditions/answer/6335093?hl=ko

[60] B. G. Gye. Possibility of AR as an educational medium and US use cases. The Department of Education. [Online]. Available: https://happyedu.moe.go.kr

[61] S. Y. Lee, S. H. Park, Y. H. Kim, S. k. Lee, and J. W. Kim, "The mobile educational contents using augmented reality," in Proc. the Korean Society of Computer Information Conference, vol. 23, no. 2, pp 212-214, 2015

[62] S. H. Jang and B. K. Kye, "Educational application of augmented reality content," The Korea Contents Association Review, vol. 5, no. 2, pp. 79-85, 2017.

[63] E. H. Park and J. W. Jeon, "Developing Korean learning contents using augmented reality," Journal of the Korea Contents Association, vol. 13 no. 4 , pp. $459-468,2013$.

[64] J. Y. Seong. (28 September 2019). Daewoo E \& C to upgrade augmented reality experience service in Prugio complex. Newdaily. [Online]. Available: http://biz.newdaily.co.kr/site/data/html/2019/09/ 28/2019092800004.html

[65] H. G. Ahn. (20 October 2019). Why is AR / VR proliferation difficult in the education market? Inews24. [Online]. Available:http://www.inews24.com/view/1215956

[66] B. K. Moon. (15 September 2019). Digital textbooks, dinosaurs, can't make a sound. Change the standard of analog review. Etnews. [Online] Available: http://m.etnews.com/20190915000048

[67] J. Lee. (18 November 2019). Difficult to build platform and burden on development cost. Chosun Daily News. [Online]. Available: https://news.chosun.com/site/data/html_dir/2019/11/17/20191117006 04.htm

[68] J. B. Kim. (11 June 2019). Korea, 4th industrial revolution environment 'worst'. Blockchain is a 'Lowest'. Inc. Block Media. [Online]. Available: https://www.blockmedia.co.kr/archives/93867
[69] S. M Park. (22 November 2019). The upcoming VR - AR era, problems to be solved. Sisaweek. [Online]. Available: http://www.sisaweek.com/news/articleView.html?idxno=128283

[70] J. Y. Kim. (19 November 2018). Brain fraud VR...What are the side effects. Moneytoday. [Online]. Available: https://news.mt.co.kr/mtview.php?no=2018110509301101439

[71] Y. J. Choi. (18 October 2019). Ministry of education, elementary school VR limited. Chosun Edu. [Online]. Available: http://edu.chosun.com/m/view.html?contid=2019101802323

Copyright $\odot 2020$ by the authors. This is an open access article distributed under the Creative Commons Attribution License which permits unrestricted use, distribution, and reproduction in any medium, provided the original work is properly cited (CC BY 4.0).

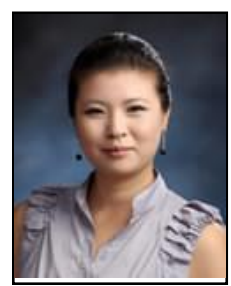

Mi-Young An was born in Incheon, S-Korea in 1971. She received her bachelor of education in 2003 from Salesian Pontifical University, Rome, and her master of education administration and doctor of education in Sociology of education from Salesian Pontifical University, Rome, Italy in 2004 and 2010, respectively. She is an associate professor in the Department of Early Childhood Education at Cheju Halla University, Jeju Special Self-Governing Province, S-Korea. She has a wide range of research interests, which include futures thinking, educational change, globalization, teaching methods and early childhood education. Her research interests in recent years focus on the integration of futures and design thinking - particularly in engineering education. Prof. An is a current member of Korean Educational Research Association, the Korean Society for Sociology of Education, the Korean Association of General Education, and the Korean Association for Learner-centered Curriculum and Instruction. She is also active members of Korea Academy-Industrial Cooperation Society, Digital Contents Society, and Korean Information Electron Communication Technology.

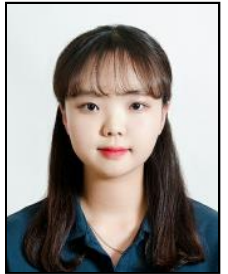

Han-Seul Kim was born in Jeju, S-Korea in 1998. She received the professional bachelor of the early childhood education in February 2019 from Cheju Halla University, Jeju, S-Korea and She has acquired the $2^{\circ}$ kindergarten instructor from Ministry of Science, Education and Technology and the 2 childcare provider from Ministry of Families and Social Welfare. She will be received the bachelor of the early childhood education in February 2020 from Cheju Halla University, Jeju, S-Korea. She worked in the elementary care class at Oedo Elementary School from March 4, 2019 to August 12, 2019.

Currently, she is interested in educational method and educational engineering and would like to study ICT utilization education in the future.

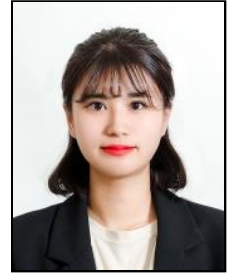

Ji-Won Kang was born in Jeju, S-Korea in 1998. She received the professional bachelor of the early childhood education in February 2019 from Cheju Halla University, Jeju, S-Korea and She has acquired the $2^{\circ}$ kindergarten instructor from Ministry of Science, Education and Technology and the 2 childcare provider from Ministry of Families and Social Welfare. She will be received the bachelor of the early childhood education in February 2020 from Cheju Halla University, Jeju, S-Korea.

She has been working at Jeju Caritas Kindergarten since April 16, 2019. Currently, she is interested in new educational methods in the 21 st century and wants to study the effects of new education for students. 